\title{
Marginal costs of hospital-acquired conditions: information for priority-setting for patient safety programmes and research
}

\author{
Authors: \\ Terri Jackson \\ Hong Son Nghiem \\ David Rowell \\ Christine Jorm \\ John Wakefield
}

Corresponding Author: terri.jackson@unimelb.edu.au

\section{Citation:}

Jackson, T., Nghiem, H.S., Rowell, D., Jorm, C. \& J. Wakefield (2011) 'Marginal costs of hospital acquired diagnoses: Information for priority setting for patient safety programs and research,' Journal of Health Services Research \&Policy, 16(3), 141-146

\section{Acknowledgements:}

The authors are grateful to the Victorian Department of Human Services and Queensland Health for access to de-identified data on individual episode costs; to the Australian Commission on Safety and Quality in Health Care and the National Health and Medical Research Council for funding; and to Ms Jude Michel for advice on coding conventions and use of ICD-10-AM. 


\section{Abstract}

Objective: To use cost data to estimate relative inpatient costs of hospital-acquired diagnoses.

Methods: Patient level costs are estimated using computerised costing systems that log individual utilization of inpatient services, and apply sophisticated cost estimates from the hospital's general ledger. Occurrence of a hospital-acquired diagnosis is identified using an Australian 'condition-onset' flag for diagnoses not present on admission. These are grouped to yield a comprehensive set of 144 categories of hospital-acquired conditions to summarise data coded with ICD-10. Standard linear regression techniques are used to identify the independent contribution of hospital-acquired conditions to costs, taking into account the casemix of a sample of acute inpatients $(n=1,699,997)$ treated in Australian public hospitals in Victoria (2005/06) and Queensland (2006/07).

Results: The most costly types of complications were post-procedure endocrine/metabolic disorders, adding (\$A) 21,869 to the cost of an episode, followed by MRSA $(19,892)$ and enterocolitis due to Clostridium difficile $(19,745)$. Aggregate costs to the system, however, were highest for septicaemia ( 41.5 mil), complications of cardiac and vascular implants other than septicaemia $(28.7 \mathrm{mil})$, acute lower respiratory infections, including influenza and pneumonia $(28.0 \mathrm{mil})$ and UTI $(24.7 \mathrm{mil})$. Hospital acquired complications are estimated to add $17.1 \%$ to treatment costs in this sample.

Conclusions: Patient safety efforts frequently focus on dramatic but rare complications with very serious patient harm. Previous studies of the costs of adverse events have provided information on 'indicators' of safety problems rather than the full range of hospital acquired conditions. Adding a cost dimension to priority-setting could result in changes to the focus of patient safety programs and research. Financial information should be combined with information on patient outcomes to allow for cost-utility evaluation of future interventions. 


\section{Introduction}

Priority-setting for patient safety programmes needs to take multiple factors into account: the frequency of the events leading to patient harm, the severity of outcomes, the absolute numbers of patients harmed, the evidence-base for the success of preventive efforts, the feasibility and acceptability of such interventions, and of course, the economic losses due to these events. One large contributor to economic loss is the additional costs of hospital care attributable to adverse events, over and above the costs of treating the patient's diagnoses on admission. ${ }^{1}$

Most efforts to use hospital administrative data to characterise patient complications have been premised on public reporting. This expectation has led to considerable investment in risk-adjustment approaches ${ }^{2-4}$ and to focussing research effort on complications deemed to be preventable. ${ }^{5}$ Using these tools, which focus on predetermined conditions of interest, it is not possible to estimate the relative contribution of all hospital-acquired illness and injury. Yet, estimation of 'preventability' changes, as new scientific evidence of causal factors emerges, together with new research on effective prevention.

Zhan and Miller's landmark 2003 study estimated additional costs of 18 types of 'medical injury'. More recently, Shreve et al. have published estimates of the costs of 50 classes of 'medical error', building on Layde et al.'s earlier classification of inpatient events. These US studies rely on billed charges to quantify costs, and did not have access to timing information to distinguish pre-hospitalisation from inpatient events, relying instead on sets of 'external cause of injury codes' to identify adverse outcomes.

Two Australian studies have attempted to quantify the costs of hospital-acquired conditions. The first ${ }^{6}$ applied DRG cost-weights to a sample of patient records from the Quality in Australian Health Care study ${ }^{7}$ to estimate the costs of adverse events. The second, and more recent study, ${ }^{8}$ used costing data from a single Australian state public hospital system to estimate the additional costs of adverse events.

The weaknesses of this study in setting priorities for prevention programmes, however, are that it resorted to ad hoc groupings of hospital-acquired diagnosis codes, considered only the first-recorded diagnosis of an in-hospital complication, and used cost data from only one State (Victoria). The recently reported Classification of Hospital Acquired Diagnoses $(\mathrm{CHADx})^{9}$ allows us to characterise complications of hospital care in a systematic and reproducible way. This categorisation contains 17 Major CHADx classes, and 144 more detailed and comprehensive subclasses, using a condition onset flag to distinguish comorbidities from conditions arising in hospital. In this paper we describe 
the use of the CHADx to estimate the per-case and total financial burdens of inpatient complications to the public hospital systems of two Australian states, representing nearly $40 \%$ of the Australian population.

\section{Methods}

Design: We use secondary analysis of routine hospital data on patient-level diagnoses, procedures and costs.

Sample: Table 1 describes the de-identified patient-level data on the 1.7 million costed public hospital episodes analysed.

\section{Table 1: Description of data sets}

\begin{tabular}{|c|c|c|c|}
\hline & Victoria & Queensland & Total \\
\hline Financial year data collection & $2005 / 06$ & $2006 / 07$ & \\
\hline $\mathrm{N}$ of hospitals & 45 & 23 & 68 \\
\hline $\mathrm{N}$ of episodes analysed & $1,083,844$ & 616,153 & $1,699,997$ \\
\hline$\%$ of sample & $64 \%$ & $36 \%$ & 100.00 \\
\hline Total recorded costs & $\$ A 3,454 \mathrm{mil}$ & $\$ A 1,899 \mathrm{mil}$ & $\$ A 5,353 \mathrm{mil}$ \\
\hline Mean cost per case & $\$ A 3,187$ & $\$ A 3,082$ & $\$ A 3,149$ \\
\hline Mean length of stay (days) & 2.95 & 2.99 & 2.97 \\
\hline \% Same-day admissions & 69.98 & 65.11 & 68.22 \\
\hline Diagnosis fields available & 40 & 50 & 50 \\
\hline Mean coding depth & 3.43 & 3.79 & 3.56 \\
\hline Incidence of any CHADx & $126,656(11.68 \%)$ & $82,122(13.32 \%)$ & $208,778(12.28 \%)$ \\
\hline $\begin{array}{l}\text { Mean of CHADx per record } \\
\text { Mean of CHADx in records with any } \\
\text { recorded complication }\end{array}$ & 0.23 & 0.19 & 0.22 \\
\hline
\end{tabular}

The mean of CHADx per record is calculated as the ratio of number of CHADx to the number of episodes. Since there are many episodes in which a patient suffered more than one CHADx (see average CHADx per complication episode in the next line), the mean of CHADx per record is larger than the incidence of CHADx.

\section{Research Variables:}

Diagnosis Codes:

Diagnosis data are abstracted from patient records by trained professional coders or health information managers; we truncated the records at 50 diagnosis fields. In both states, data are routinely edited by the respective health departments against published standards for plausible code combinations, and both states conduct audits of patient records from public hospitals. ${ }^{10,11}$ In Victoria, financial penalties are applied for funded 
hospitals with exceptional rates of coding error. ${ }^{11}$ National coding standards require that all diagnoses monitored or treated during the admission be recorded. ${ }^{12}$

\section{Diagnosis Onset Flag:}

Victorian coding has included information on the timing of diagnoses since at least 1980. For application of a ' $C$ ' prefix (indicating a 'complication') the coder must ascertain that there was no evidence of the condition existing prior to admission, that is, the C-prefix is used only for a diagnosis arising after admission. Queensland adopted a similar numeric 'diagnosis onset flag' in July 2006. Both distinguish co-morbidities from hospital-acquired diagnoses, and incident diagnoses (arising during the current episode) from those in readmission episodes. ${ }^{13}$ The more recent adoption of onset-flagging by Queensland meant that only one year (2006/07) of data was available. Flagging was evaluated using a recently developed algorithm to identify chronic and congenital conditions that are unlikely to be hospital-acquired. ${ }^{14}$

\section{Total Cost:}

State health authorities in both Victoria and Queensland collect annual patient-level costing datasets from public hospitals in their respective states. Costs are estimated using computerised clinical costing systems that identify the costs of hospital care for individual patients. ${ }^{15}$ The 45 hospitals contributing to the dataset from Victoria are larger urban hospitals, accounting for $86.4 \%$ of weighted inpatient activity (i.e., weighted according to complexity) for the period 1 July 2005-30 June 2006. These costs are subjected to data quality checks before calculation of cost weights for casemixbased hospital funding, including re-submission of cases with missing data to the originating hospital. ${ }^{15}$

A later dataset was requested from Queensland Health because it was the second state in Australia to flag hospital-acquired diagnoses, beginning only in the 2006/2007 FY. The Queensland cost data are collected from all hospitals in the state for submission to the National Hospital Cost Data Collection (NHCDC). In this study, we use only those costed episodes evaluated and accepted for inclusion in the National dataset $(n=616,153$ cases in 23 hospitals).

Cost per case includes the costs of treating the primary diagnosis (reason for admission), any additional costs attributable to 'present on admission' diagnoses (co-morbidities), and the costs of any hospital-acquired diagnoses (complications). 
Uncomplicated Treatment Cost: that portion of each patient's measured cost per case attributable to their principal diagnosis and co-morbidities. This is calculated for each DRG as the mean of all episodes with no Hospital Acquired Diagnosis (CHADx).

Mean Corrected Treatment Cost: that portion of each patient's measured cost per case remaining after subtracting the Uncomplicated Treatment Cost for the DRG assigned to the episode. These costs are used as the dependent variable in linear regression analyses to estimate the marginal effects of each CHADx.

Total Cost: a calculation of the costs attributable to a particular CHADx based on the marginal CHADx Treatment Cost multiplied by the number of instances of the CHADx in the study database.

Sameday patients: a dummy variable, equal to one for patients treated in hospital on a 'sameday' basis, and zero for multi-day patients. The Australian DRG version, ARDRGs, ${ }^{16}$ incorporates all inpatient episodes, including sameday surgery and other procedures classed as 'ambulatory' in other classification systems.

Death in Hospital: a dummy variable equal to one if the patient dies in hospital, and zero otherwise, to control for any costs associated with end-of-life treatment that might distort estimates of costs for some hospital-acquired conditions.

\section{Analysis}

Standard linear regression was used to investigate the relationship between the cost of hospitalisation and adverse events, after controlling for other covariates. Specification tests were applied to verify the choice of variables, and a heteroskedasticity test was applied to test the assumption of constant variance in regression analyses.

Since our data represent a relatively large sample (1.7 million observations), standard regressions can be used to produce robust and consistent results in spite of the fact that the cost data may not satisfy the normality assumption. ${ }^{17}$ Untransformed data allow the expression of results in easily-interpretable dollar terms.

\section{Results}

Table 2 shows the full results from our OLS model. As expected, the majority of CHADx dummy variables have positive and significant parameter estimates, suggesting that they consistently add cost compared with uncomplicated episodes in the same DRG. The costs of same-day episodes and those episodes where the patient dies in hospitals are significantly lower compared with other episodes. Our model achieved an $\mathrm{R}^{2}$ of 0.26 . 


\section{Table 2: Complete OLS estimates for costs not attributable to treatment of an uncomplicated case in the same DRG.}

Intercept

Died in hospital

$-\$ 1,139$

Sameday

$1,159,712$

$-\$ 57$

CHADx 1_1

Complications of infusion /transfusion

690

$\$ 1,847$

CHADx 1_2

Gas embolism

$6 \$ 2,186$

CHADx 1_3

Failed or difficult intubation

433

$\$ 12$

CHADx 1_4

Other haemorrhage \& haematoma complicating a procedure

5,064

$\$ 2,885$

CHADx 1_5

Accidental puncture/lac during proc

1,991

$\$ 1,640$

CHADx 1_6

Foreign body or substance left following procedure

40

$\$ 5,215$

CHADx 1_7

Other comps of surgical and medical NEC (Incl shock T81.1)

CHADx 1_8

Disruption of wound

1,543

$\$ 3,688$

CHADx 1_9

Wound infection (exc septicaemia)

1,094

$\$ 9,515$

CHADx 1_10

Complications of cardiac and vascular implants (exc septicaemia)

2,627

$\$ 4,528$

CHADX 1_11

Complications of genitourinary implants (exc septicaemia)

3,702

$\$ 7,743$

CHADX 1_12

Complications of orthopaedic implants (exc septicaemia)

958

$\$ 4,165$

CHADx 1_13

Complications of other implants (exc septicaemia)

CHADx 1_14

Complications of transplants

291

$\$ 11,982$

1,214

$\$ 9,843$

CHADx 1_15

Complications of reattachment and amputations

285

$\$ 7,092$

CHADx 1_16

Post-procedural disorders: endocrine \& metabolic

115

$\$ 8,888$

CHADx 1_17

Post-procedural disorders: nervous system

46

$\$ 21,869$

CHADx 1_18

Post-procedural disorders: eye \& ear

389

$\$ 6,163$

CHADx 1_19

Post-procedural disorders: circulatory system

119

$\$ 743$

CHADx 1_20

Post-procedural disorders: respiratory system

1,947

$\$ 2,698$

Post-procedural disorders: digestive system

CHADx 1_21

Post-procedural disorders: musculoskeletal system

1,558

$\$ 6,727$

CHADx 1_22

Post-procedural disorders: genitourinary system

1,566

$\$ 6,185$

CHADx 1_23

CHADx 2_1

Skin Adverse effects dt systemic antibiotics

98

$\$ 2,502$

$882 \$ 2,037$

$741 \$ 4,075$

CHADX 2_2

Other adverse effects dt systemic antibiotics

CHADx 2_3

Nausea \& vomiting dt antineoplastic drugs

1,129

$\$ 6,729$

239

$\$ 174$

CHADx 2_4

Other adverse effects dt antineoplastic drugs

$514 \$ 1,413$

Coagulation defect dt drugs affecting blood constituents

CHADx 2_5

Other adverse effects dt drugs affecting blood constituents

CHADx 2_6

Nausea and vomiting dt opioids and related analgesics

Alterations to mental state dt opioids and related analgesics

CHADx 2_8

CHADx 2_9 Other adverse effects dt opioids and related analgesics

$919 \$ 3,021$

$554 \$ 4,896$

$212 \$ 1,614$

$417 \$ \$ 679$

$858 \$ 1,918$ 
CHADx 2_10

CHADx 2_11

CHADx 2_12

CHADX 2_13

CHADx 2_14

CHADx 2_15

CHADx 2_16

CHADx 2_17

CHADx 2_18

CHADx 3_1

CHADx 3_2

CHADx 3_3

CHADx 3_4

CHADx 3_5

CHADx 4_1

CHADx 4_2

CHADx 4_3

CHADx 4_4

CHADx 4_5

CHADx 5_1

CHADx 5_2

CHADx 5_3

CHADx 5_4

CHADx 5_5

CHADx 5_6

CHADx 5_7

CHADx 5_8

CHADx 5_9

CHADx 5_10

CHADx 5_11

CHADx 6_1

CHADx 6_2

CHADx 6_3

CHADx 6_4

CHADx 6_5

CHADx 6_6

CHADx 6_7

CHADx 6_8
Adverse effects dt anaesthesia (Incl misadventure)

Hypotension dt anaesthesia

Alterations to mental state $\mathrm{dt}$ anaesthesia

Other adverse effects dt drugs affecting $C-V$ system

Hypotension dt drugs affecting $C-V$ system

Adverse effects dt insulin \& oral hypoglycaemics

Adverse effects dt other drugs

Anaphylactic shock dt correct drug properly administered

Incorrect drug dosage/ combination administered

Falls with fractured neck of femur

Falls with intracranial injury

All other falls

Injury $\mathrm{d} / \mathrm{t}$ assault

Other patient accidents (exc poisoning)

Septicaemia

Mycoses

MRSA

Other drug resistant infections

Other infectious agents

AMI

Pulmonary embolism (PE)

Cardiac arrythmias, conduction disturbances \& abnormal heart beat

Ventricular fibrillation/ cardiac arrest

Heart failure

Hypotension (not drug induced)

Cerebro-vascular disease \& TIA

Venous thrombosis/embolism (not progressing to PE)

Unstable and other angina

Cardiogenic and other shock

Other circulatory system complications

ARDS, respiratory failure \& pulmonary collapse (incl atelectasis)

Aspiration pneumonia

Acute lower respiratory infections (incl influenza \& pneumonia)

Pulmonary oedema, pneumothorax \& pleural effusion

Haemorrhage from respiratory passages

Asphyxia \& respiratory arrest

Breathing difficulties

Other hospital-acquired respiratory disorders

\begin{tabular}{rr}
532 & $-\$ 1,562$ \\
133 & $\$ 1,353$ \\
102 & $\$ 1,434$ \\
708 & $\$ 925$ \\
328 & $\$ 1,024$ \\
39 & $\$ 5,103$ \\
3,954 & $\$ 3,217$ \\
89 & $-\$ 4,044$ \\
256 & $\$ 5,245$ \\
124 & $\$ 12,002$ \\
58 & $\$ 2,052$ \\
2,347 & $\$ 3,910$ \\
51 & $\$ 8,953$ \\
1,403 & $\$ 5,193$ \\
4,406 & $\$ 9,420$ \\
2,390 & $\$ 6,034$ \\
352 & $\$ 19,892$ \\
978 & $\$ 12,298$ \\
742 & $\$ 8,004$ \\
1,429 & $\$ 1,874$ \\
1,601 & $\$ 7,503$ \\
2,201 & $\$ 833$ \\
485 & $\$ 1,918$ \\
1,045 & $\$ 3,388$ \\
1,136 & $\$ 4,174$ \\
5,064 & $\$ 5,517$ \\
3,122 & $\$ 5,118$ \\
1,590 & $-\$ 347$ \\
2,891 & $-\$ 128$ \\
10,551 & $\$ 1,085$ \\
1,093 & $\$ 4,237$ \\
1,209 & $\$ 6,991$ \\
598 & $\$ 1,777$ \\
400 & $-\$ 2,585$ \\
966 & $\$ 6,690$ \\
\hline 3,265 \\
\hline 347
\end{tabular}


CHADx 7_1

CHADx 7_2

CHADx 7_3

CHADx 7_4

CHADx 7_5

CHADx 7_6

CHADx 7_7

CHADx 8_1

CHADx 8_2

CHADx 8_3

CHADx 8_4

CHADx 9_1

CHADX 9_2

CHADx 9_3

CHADx 9_4

CHADx 9_5

CHADx 10_1

CHADx 10_2

CHADx 10_3

CHADX 10_4

CHADx 10_5

CHADx 10_6

CHADx 11_1

CHADx 12_1

CHADx 12_2

CHADx 12_3

CHADX 12_4

CHADx 12_5

CHADX 12_6

CHADx 12_7

CHADx 12_8

CHADX 12_9

CHADx 12_10

CHADx 12_11

CHADx 12_12

CHADx 12_13

CHADx 12_14
Gastro enteritis

Paralytic ileus \& intestinal obstruction (w/o hernia)

Enterocolitis dt Clostridium difficile

Constipation

Nausea and vomiting

GI bleeding not classified to a disease

Other digestive system disorders

Pressure ulcers

Cellullitis

Dermatitis, rash \& other skin effects

Other skin disorders

Acute \& unspecified renal failure (exc post procedural)

UTIs

Urinary retention

Other complications \& symptoms of the urinary system

Other complications of male \& female genitals

Depressive episode \& symptoms involving emotional state

Panic and other anxiety disorders

Adjustment \& other psych disorders

Alterations to mental state

Mental \& behavioural disorders due to psychoactive substance use

Patient self-harm (Incl intentional and undetermined intent overdose)

Complications of abortion, ectopic and molar pregnancies

Foetal heart rate abnomalies

Foetal meconium and other distress

Complications of umbilical cord

Unsuccessful Interventions during labour

Complications of maternal anaesthetic

First degree and unspecified perineal laceration

Second degree perineal laceration

Third degree and fourth degree perineal laceration

Maternal haemorrhage

Other obstetric injury

Other complications intrapartum \& postpartum

Retained placenta

Maternal infection (exc wound infection \& septicaemia)

Breast disorders associated with childbirth
$4,592 \$ \$ 4,700$

$1,195 \quad \$ 4,087$

$233 \$ 19,745$

$5,749 \quad \$ 3,312$

$6,744 \quad \$ 440$

$1,804 \$ 4,211$

$3,065 \$ 6,876$

$2,873 \quad \$ 8,461$

$997 \$ 2,749$

$5,468 \quad \$ 2,920$

$1,208 \$ \$ 5,366$

$3,608 \quad \$ 2,550$

$6,714 \quad \$ 3,675$

$3,898 \quad-\$ 43$

$4,706 \quad \$ 787$

$741 \$ 3,375$

$2,252 \quad \$ 3,141$

$743 \$ 3,425$

$450 \$ 6,157$

$5,442 \quad \$ 2,062$

$597 \quad \$ 114$

$262 \$ 6,953$

$3,768 \quad \$ 342$

$4,939 \quad \$ 145$

$3,831 \quad-\$ 14$

$8,323-\$ 202$

$1,657 \quad \$ 405$

$436-\$ 1,357$

$4,657 \quad-\$ 139$

$13,098 \quad-\$ 114$

$1,166 \quad-\$ 604$

$11,556 \quad \$ 170$

$4,198 \quad-\$ 525$

$5,302 \quad \$ 19$

$969 \quad-\$ 229$

$1,156 \$ \$ 1,032$

$11,184 \quad-\$ 108$ 
CHADx 12_15

CHADx 13_1

CHADx 13_2

CHADx 13_3

CHADX 13_4

CHADx 13_5

CHADx 13_6

CHADx 13_7

CHADx 13_8

CHADx 13_9

CHADx 13_10

CHADx 13_11

CHADx 14_1

CHADx 14_2

CHADx 14_3

CHADx 14_4

CHADx 15_1

CHADx 15_2

CHADx 15_3

CHADx 15_4

CHADx 15_5

CHADx 15_6

CHADx 16_1

CHADx 16_2

CHADx 16_3

CHADx 17_1

CHADx 17_2

CHADx 17_3

CHADx 17_4

CHADx 17_5

CHADx 17_6

CHADx 17_7

CHADx 17_8

CHADx 17_9

CHADx 17_10

CHADx 17_11

CHADx 17_12
Other disorders predominantly related to pregnancy

Prenatal injuries

Intracranial haemorrhage, hypoxia and other brain injuries

Other birth trauma

Respiratory distress of newborn

Aspiration \& other respiratory disorders of newborn

Circulatory disorders of newborn

Perinatal infections (exc septicaemia)

Haemorrhage and blood disorders of newborn

Jaundice

$\mathrm{Gl}$ and feeding disorders of newborn

Other neonatal complications

Post haemorrhagic anaemia (not post-procedural)

Other hospital-acquired anaemia

Coagulation defects

Agranulocytosis, thrombocytopenia \& other blood disorders

Dehydration / volume depletion

Electrolyte disorders w/o dehydration

Hospital acquired nutrition deficiencies (incl nutritional anaemia)

Hypoglycaemia \& hyperglycaemia

Disorders of mineral metabolism

SIADH, hyperthyroidism \& other metabolic disorders

Hospital-acquired paralysis

Dystonia, tremors \& gait disorders

Other nervous system complications

Major symptoms

Headache \& migraine

Oedema \& ascites

Chest pain

Abdominal pain

Fever (not classified to condition)

Convulsions

Dizziness, fainting \& blackout

Complications of the eye and ear

Musculoskeletal complications (not associated with falls)

Dysphagia

Other hospital-acquired symptoms
2,372 $-\$ 479$

2,388

$\$ 2,815$

2,164 $-\$ 116$

5,821 $-\$ 682$

3,121

$\$ 3,429$

1,938 $\$ 867$

3,332

$\$ 2,838$

1,005

$\$ 629$

6,600 $-\$ 577$

11,103 $\$ 737$

12,459 $\$ 513$

2,751

$\$ 3,118$

5,529

$\$ 2,897$

1,383

$\$ 2,874$

2,596

4,374

$\$ 6,116$

17,555 $\$ 399$

1,953

741

3,501

315

628

775

1,335

1,605

2,228

1,728

3,999

1,504

4,805

716

2,191

1,498

3,813

903

$\$ 1,316$

$\$ 3,708$

$\$ 744$

$\$ 2,273$

$\$ 7,023$

$\$ 4,093$

$\$ 144$

$\$ 10,478$

$\$ 3,257$

$\$ 1,371$

$\$ 3,973$

$\$ 1,368$

$\$ 1,662$

$\$ 1,074$

$\$ 2,354$

$\$ 621$

1,979
$\$ 4,062$

$\$ 2,596$

$\$ 1,199$

$\$ 1,482$ 
Total

Table 3 reports the ten CHADx that generate the highest additional costs per episode. These are generally low volume diagnoses with very costly consequences, the exception being septicaemia, with both high per episode costs and reasonably high volume of cases. Two of the top ten are drug resistant infections (Methicillin Resistant Staph Aureus (CHADx 4.3) and Vancomycin or other drug-resistant infections (CHADx 4.4)). These and others in the top 10, such as wound disruption/dehiscence (CHADx 1.8) and in-hospital falls resulting in hip fracture (CHADx 3.1) feature in most prevention/ patient safety programs.

Table 3: Top 10 Complications (CHADx) by additional cost per episode

\begin{tabular}{|c|c|c|c|}
\hline$C H A D x$ & Descriptor & $\begin{array}{l}N \text { of } \\
\text { cases }\end{array}$ & $\begin{array}{l}\text { Additional } \\
\text { cost per } \\
\text { episode }\end{array}$ \\
\hline 1_16 & Post-procedural disorders: endocrine \& metabolic & 46 & $\$ 21,869$ \\
\hline $4 \_3$ & MRSA & 352 & $\$ 19,892$ \\
\hline 7_3 & Enterocolitis due to Clostridium difficile & 233 & $\$ 19,745$ \\
\hline 4_4 & Other drug resistant infections & 978 & $\$ 12,298$ \\
\hline 3_1 & Falls with fractured neck of femur & 124 & $\$ 12,002$ \\
\hline 1_12 & Complications of orthopaedic implants (exc septicaemia) & 291 & $\$ 11,982$ \\
\hline $16 \_3$ & Other nervous system complications & 1,335 & $\$ 10,478$ \\
\hline 1_13 & Complications of other implants (exc septicaemia) & 1,214 & $\$ 9,843$ \\
\hline $1 \_8$ & Disruption of wound & 1,094 & $\$ 9,515$ \\
\hline 4_1 & Septicaemia & 4,406 & $\$ 9,420$ \\
\hline
\end{tabular}

Table 4 reports the top ten CHADx in terms of additional system costs (per episode by the number of instances). Septicaemia heads this list because it is both costly per episode and relatively frequent. The high volume of cases of electrolyte disorders not associated with dehydration (CHADx 15.2) results in a relatively low-cost complication (\$A1,339 per episode) appearing in the top ten in terms of total system cost impact. 
Table 4: Top 10 complications (CHADx) by total additional system cost

\begin{tabular}{|c|c|c|c|}
\hline CHADx & Descriptor & $\begin{array}{l}N \text { of } \\
\text { cases }\end{array}$ & $\begin{array}{l}\text { Additional } \\
\text { system cost }\end{array}$ \\
\hline 4_1 & Septicaemia & 4,406 & $\$ 41,505,215$ \\
\hline 1_10 & Complications of cardiac and vascular implants (exc septicaemia & 3,702 & $\$ 28,665,676$ \\
\hline $6 \_3$ & Acute lower respiratory infections & 5,064 & $\$ 27,939,311$ \\
\hline 9_2 & UTIS & 6,714 & $\$ 24,673,623$ \\
\hline 8_1 & Pressure ulcers & 2,873 & $\$ 24,307,711$ \\
\hline 15_2 & Electrolyte disorders $\mathrm{w} / \mathrm{o}$ dehydration & 17,555 & $\$ 23,095,970$ \\
\hline 7_1 & Gastro enteritis & 4,592 & $\$ 21,582,217$ \\
\hline 7_7 & Other digestive system disorders & 3,065 & $\$ 21,076,154$ \\
\hline 7_4 & Constipation & 5,749 & $\$ 19,042,144$ \\
\hline $6 \_1$ & ARDS, respiratory failure \& pulmonary collapse & 5,087 & $\$ 18,509,336$ \\
\hline
\end{tabular}

Our econometric model produced 18 negative coefficients of which 15 were statistically significant. An inspection of Table 2 shows that four of these negative coefficients were associated with anaesthesia and 11 with childbirth. It is not clear why this might be the case, although it is likely that the negative coefficients reflect complex interactions between the individual CHADx when they co-occur within the same admission. Alternatively, some CHADx may truly be cost reducing in any single episode. For example, anaesthetic complications could lead to cancellation of surgery.

We have factored the negative values into our estimates of total system costs. Although statistically significant, the low frequencies resulted in only a small effect on total cost, and we do not believe that the negative coefficients compromise our ability to interpret findings for the majority of CHADx. In total, hospital-acquired diagnoses add $\$ 782$ mil to the costs of inpatient care in the two states analysed here, representing $14.6 \%$ of total expenditures; and additional costs of $17.1 \%$.

\section{Discussion}

Patient safety programmes are usually initiated with little or no evaluation of priorities. More rational priority-setting would involve evaluation of several dimensions of the problem. Obviously, the expected benefits for patient survival and quality of life of preventing particular kinds of hospital-acquired illness and injury must be considered. Evidence about the effectiveness of potential interventions in reducing the rate of such 
complications, and the relative costs of introducing such programs must also be considered, ${ }^{18}$ and for research priorities, the expected value of perfect information.

Warburton ${ }^{21}$ has argued that patient safety interventions should meet the same costeffectiveness criteria as other medical interventions. The CHADx estimates reported here provide the basis on which the cost-effectiveness of interventions to reduce rates of these common complications could be evaluated. Expressed as an index (using the mean cost per case as denominator), our results could be extrapolated to comparable hospitals and care health systems.

This study provides for the first time estimates of the relative costliness of patient safety problems across the spectrum of patient harm, rather than for a narrower set of safety indicators. It addresses the problem highlighted by Runciman that mundane complications of care are often overlooked when priorities for patient safety interventions are evaluated. ${ }^{19,20}$ Our analysis suggests that CHADx 4.1 Septicaemia, 1.10 Complications of cardiac and vascular implants (exc septicaemia), 6.3 Acute lower respiratory infections (including influenza \& pneumonia), 9.2 Urinary Tract Infections, and 8.1 Pressure ulcers represent the highest system cost complications in our 2-state sample of costed patient episodes. Most of these complications are common, and if not preventable in every case, are amenable to reduction in their rates.

Pressure ulcers (CHADx 8.1) are already a frequent target of prevention programs because of their implications for extended length of stay, but their financial costs have perhaps not been fully appreciated. Others complications, however, are not so commonly recognised as resulting in high per-case costs including the Post-procedural endocrine disorders (CHADx 1.16), and Complications of surgical implants (CHADx 1.13). Better understanding of the financial burden of these diagnoses, can help make the 'business case' for greater investment in research on how best to prevent them, and for evidence-based interventions. ${ }^{20}$

Our findings are subject to a number of limitations. They are clearly dependent on the quality of coding and prefixing of codes in hospitals. We consider the quality assurance programs for inpatient data in the two states to be robust, but we have not undertaken verification by parallel prospective data collection or independent chart review. The Victorian cost data used were from one year prior to the Queensland data. Because the period was one of low general inflation, observed cost differences between the two health systems were small, and other sources of variation in the sample were large, we did not convert the data to a single price year. 
Our use of linear regression assumes that episodes with multiple CHADx reflect random combinations of complications. Clinical reasoning suggests, however, that some CHADx may frequently co-occur. To the extent that clustering exists, it may distort some of the coefficient-based CHADx costs reported here. We are currently undertaking work to better understand underlying 'syndromes' of these diagnoses when they co-occur, particularly in maternity episodes.

The cost data used in the study are not normally distributed, perhaps leading to concern that our analysis strategy should have involved log transformation of the data. We note that normality is a sufficient but not a necessary condition for application of ordinary least squares regression, particularly when samples are sufficiently large. Lumley et al. support this strategy, demonstrating that samples with less than 500 observations can provide unbiased estimates. ${ }^{18}$ We believe that our data, which contains approximately 1.7 million observations, comfortably satisfies this criterion, and that policy uses of the findings are better served by interpretable cost estimates.

Earlier models tested in this study included a term for patient age and higher order polynomials of age as alternatives to a linear specification of the relationship of age and costs. While age variables were found to be significant predictors of increased treatment cost (taking the DRG into account), we have omitted them from the final model for policy reasons. It is certainly true that older patients are more vulnerable to hospital-acquired conditions. ${ }^{22}$ If CHADx costs were to be adjusted for the patient's age through inclusion in the model, this would reduce the cost estimates for any CHADx that disproportionately affects older people, and perhaps their priority for quality improvement efforts. Of course, it would be possible to re-weight each CHADx treatment cost using the appropriate coefficients for the distribution of patients' age in the CHADx, but this is more cumbersome than simply leaving any age-related additional costs in the CHADx coefficient.

Patient safety efforts frequently focus on dramatic but rare complications with very serious patient harm. Useful information to reduce morbidity across the severity spectrum requires a comprehensive list of events ('preventable' or not with current clinical knowledge). Adding an economic dimension to priority-setting could result in changes to the priorities of patient safety programs, and would allow for cost-utility evaluation of existing prevention programs. Such information may suggest future research directions, including study of causes and solutions for 'mundane' adverse events that cause patient harm and increase inpatient costs. 


\section{References}

1. Zhan C, Miller MR. Excess length of stay, charges, and mortality attributable to medical injuries during hospitalization. JAMA. 2003;290:1868-74.

2. Iezzoni LI, Daley J, Heeren T, et al. Identifying complications of care using administrative data. Med Care. 1994;32:700-15.

3. McCarthy EP, lezzoni LI, Davis RB, et al. Does clinical evidence support ICD-9-CM diagnosis coding of complications? Med Care. 2000;38:868-76.

4. Weingart SN, lezzoni LI, Davis RB, et al. Use of administrative data to find substandard care: Validation of the complications screening program. Med Care. 2000; 38:796-806.

5. Hughes JS, Averill RF, Goldfield NI, et al. Identifying potentially preventable pomplications using a present on admission indicator. Health Care Financ Rev. 2006;27:63-82.

6. Rigby K, Clark RB, Runciman WB. Adverse events in health care: setting priorities based on economic evaluation. J Qual Clin Pract. 1999;19:7-12.

7. Wilson R, Runciman W, Gibberd R, et al. The quality in Australian health care study. Med J Aust. 1995;163:458-71.

8. Ehsani J, Jackson TJ, Duckett S. The incidence and cost of adverse events in Victorian hospitals, 2003-04. Med J Aust. 2006; 184:551-5.

9. Jackson T, Michel J, Roberts R, et al. 'A classification of hospital acquired diagnoses for use with routine hospital data'. Med J Aust, 2009; 191:544-548.

10. Logan E, Turtle J, Wiseman S. Coding audits - a positive experience. Health Information Management Journal. 2006;35:36-40.

11. Henderson T, Shepheard J, Sundararajan V. Quality of diagnosis and procedure coding in ICD-10 administrative data. Med Care. 2006; 44:1011-9.

12. Health Data Standards and Systems Unit. Victorian Additions to Australian Coding Standards: Vic 0002 Additional diagnoses. Victoria: Victorian State Government, Department of Human Services, Australia; 2006 [accessed 25 Mar 2010]; Available from: http://www. health.vic.gov.au/hdss/icdcoding/vicadditions/vicadd6.pdf.

13. Jackson TJ, Duckett SJ, Shepheard J, et al. Measurements of adverse events using 'incidence flagged' diagnosis codes. J Health Serv Res Policy 2006;11:21-6.

14. Jackson TJ, Michel J, Roberts R, et al. Development of a validation algorithm for 'condition onset' flagging. BMC Medical Informatics and Decision-Making, 2009; 9:48.

15. Jackson T. Cost estimates for hospital inpatient care in Australia: evaluation of alternative sources. Aust N Z J Public Health. 2000;24:234-41.

16. Department of Health and Ageing. Australian Refined Diagnosis Related Groups Version 5.0. Canberra: Commonwealth Department of Health and Ageing, 2002.

17. Lumley T, Diehr P, Emerson S, et al. The importance of the normality assumption in large public health data sets Annu Rev Public Health. 2002;23:151. 
18. Jackson TJ. One Dollar in Seven: Scoping the Economics of Patient Safety. Report to the Canadian Patient Safety Institute, September 2009. Accessed 24/08/2010 at:

http://www.patientsafetyinstitute.ca/English/research/commissionedResearch/E conomicsofPatientSafety/Documents/Economics of Patient Safety Literature Review.pdf

19. Runciman WB, Edmonds MJ, Pradhan M. Setting priorities for patient safety. Qual Saf Health Care. 2002;11:224-9.

20. Dimick J, Weeks W, Karia R, et al. Who pays for poor surgical quality? Building a business case for quality improvement. J Am Coll Surg. 2002;202:933-7.

21. Warburton RN. Patient safety--how much is enough? Health Policy. 2005;71:22332.

22. Rowell D, Nghiem HS, Jorm C, Jackson TJ .How different are inpatient complications in the elderly?' forthcoming 2010; accepted for publication in Quality and Safety in Health Care (August, 2009). 